\title{
XIV.
}

\section{Manometrische Druckbestimmungen an einer äusseren Lungenfistel des Menschen.}

\author{
Von Prof. Dr. Hermann Eichhorst \\ in Zürich.
}

So überraschend oft man auch Verwachsungen zwischen Lungen und Brustwand zu sehen bekommt, so ausserordentlich selten greifen unter solchen Umständen erfahrungsgemäss entzündliche und ulcerative Veränderungen der Lungensubstanz auf die Brustwand selbst über. Wie viele Aerzte giebt es, welche Zeit ihres Lebens Tausende und Abertausende von Lungenkranken behandelt haben, ohne jemals einem solchen Ereigniss gegenübergestanden zu haben! Mir selbst sind derartige Dinge trotz eines seit Jahren sehr ausgedehnten Beobachtungskreises nur zwei Male begegnet, und nach den spärlichen Aufzeichnungen in der Literatur sollte man fast meinen, dass ich dabei durch einen besonderen Glückszufall begünstigt worden bin.

Vor etwas über 10 Jahren behandelte ich eine Lungenschwindsüchtige, bei welcher sich während einer Nacht ungefähr in der Mitte des zweiten linken Intercostalraumes über einer $5 \mathrm{~cm}$ im Durchmesser haltenden Stelle ein unverkennbares subcutanes Emphysem entwickelt hatte. Man konnte kaum an eine andere Erklärung denken, als dass die vordem diagnosticirte Lungenhöhle durch vorhergegangene Verwachsung mit der Brustwand bis in das Unterhautzellgewebe vorgedrungen sei und sich gewissermaassen in dasselbe eröffnet habe. Als die Kranke wenige Tage später durch Entkräftung zu Grunde ging, zeigte es sich, dass die Verhältnisse so lagen, wie man sie während des Lebens vermuthet hatte. Es war also zur Ausbildung einer unvollständigen Lungen-Hautfistel gekommen.

Aehnlich, nur in einer noch weiter vorgeschrittenen Entwickelung gestalteten sich die Veränderungen in einer zweiten Beobachtung, welche mir vor wenigen Monaten auf der Züricher 
Klinik unter die Hände kam. Ein 42 jähriger Mann, welcher bisher dem Gewerbe eines Küfers obgelegen hatte, bot die Erscheinungen einer faustgrossen tuberculösen Höhle im Oberlappen der linken Lunge dar. Za gleicher Zeit fand sich nahe dem unteren Rande der ersten linken Rippe eine Fistelöffnung, aus welcher'sich in mässiger Menge eine durch umgesetzten Blutfarbstoff hellbräunlich-roth gefärbte Flüssigkeit entleerte. Sobald der Kranke mit gelinder Gewalt hustete, trat aus dieser Fistel ausserdem unter einem weithin hörbaren zischenden Geräusch Luft heraus, welche nicht selten die kleinen Eitermengen in Gestalt grosser Luftblasen mit sich heraustrieb. Es liess sich nun, wie im Folgenden genauer gezeigt werden wird, mit Sicherheit der Beweis liefern, dass die in diesem Falle vollständige Lungenhautfistel einen ventilartigen Bau besitzen musste, so dass zwar Luft aus der Lunge heraus-, aber nicht umgekehrt Luft von aussen durch die Fistel in die Lunge hineindringen konnte. Zu gleicher Zeit schien bei diesem Kranken die Möglichkeit gegeben, mit Hülfe eines in die Fistelöffnung gesetzten Manometers die Grösse des exspiratorischen Luftdruckes zu messen. Man wird sich ja begreiflicherweise niemals der geringsten Täuschung darüber hingeben wollen, dass in unserer Beobachtung die Verhältnisse für derartige Druckmessungen keineswegs die denkbar günstigsten waren, aber da bisher überhaupt noch niemals derartige Druckmessungen ausgeführt worden sind, wird man es begreiflich finden, wenn wir unsere Erfahrungen trotz ihrer Mängel und Lückenhaftigkeit dennoch zur Veröffentlichung bringen. Um ein richtiges Urtheil ïber unser Unterfangen gewinnen zu können, erscheint es nothwendig, die. Hauptthatsachen aus der Krankengeschichte wiederzugeben.

Beobachtung.

Ernst Fischer, 42 Jahre alt, Küfer aus Bärentsweil, stammt aus einer Familie, in welcher wohl zweifellos tuberculöse Erkrankungen vorgekommen sind. Sein Vater ging durch ein langsam fortschreitendes Lugenleiden zu Grunde und einer seiner Brüder litt. viele Jahre an Knochenfrass.

Patient ist wiederholentlich in dem Züricher Cantonsspital behandelt worden. Mit Ausnahme von hartnäckigen Augenentzündungen will er in seimer Jugend immer gesund gewesen sein. Vor länger als zwanzig Jahren zog er sich eine starke Anschwellung eines Armes za, um deretwillen er einige Wochen auf der chirurgischen Abtheilung des Cantonsspitales be- 
handelt wurde. Im Jahre 1877 stellten sich, angeblich ohne vorhergegangene Geschlechtskrankheit, Lymphdrüsenanschwellungen in beiden Inguinalbeugen ein. Der Kranke liess sich deswegen auf die medicinische Abtheilung des Cantonsspitales aufnehmen. Die Drüsen kamen hier allmählich zur Vereiterung, so dass man auf beiden Seiten mehrfache Einschnitte machen musste. Die ärztliche Behandlung, während welcher Patient fast ununterbrochen das Bett büten musste, dauerte volle vier Monate.

Im Jahre 1879 wiederholte sich die Erkrankung und so brachte Patient wieder drei Monate auf der medicinischen Klinik des Cantonsspitales zu.

Seit vier Jahren leidet Patient an Husten und zunehmender Schwäche. Er wurde deswegen rom 31. Oetober bis zum 26. November 1888 in Absonderungshaus der medicinischen Klinik auf der Phthisikerabtheilung behandelt. Der wesentlichste Befund beschränkte sich damals auf eine Dämpfung in der linken Supraclaviculargrube und Regio suprascapularis. Dabei stand die linke Lungenspitze knapp $1 \mathrm{~cm}$ weniger hoch als die rechte. Das Athmungsgeräusch war sehr leise und unbestimmt. Rasselgeräusche liessen sich nicht wahrnehmen. Der Kranke warf nur sehr geringe Mengen schaumiger Massen aus, in welchen niemals Tuberkelbacillen nachgewiesen werden konnten. Ausser einer kräftigenden Diät verordnete man Leberthran und Pillen aus Ferrum lacticum, Kalium bromatum und Acidum arsenicosum. Der Kranke, welcher übrigens nie fieberte, erholte sich sehr schnell und nahm in vier Wochen um $2 \frac{1}{4} \mathrm{~kg}$ Körpergewicht $\mathrm{zu}$. Er fühlte sich wieder vollkommen frei von Beschwerden und zugleich so kräftig, dass er selbst seinen Austritt aus dem Spital verlangte, um wieder seiner Arbeit und dem Verdienste nachzugehen.

Am 20. Januar 1882 meldete sich der Kranke von Neuem zur Aufnahme auf die Klinik und brachte hier die Zeit bis zum 29. Februar 1.892 zu. Sein Zustand hatte sich mittlerweile sehr beträchtlich verschlimmert, was er auf eine Verletzung zurückführt, welche er vor etwa 6 Monaten erlitt. Um diese Zeit stiess er mit der Vorderfäche seines linken Brustkorbes heftig gegen ein Fass, wobei er sich die zweite linke Rippe gebrochen. haben will. Patient suchte sich erst selbst mit Pflastern und Geschmiersel mannichfaltiger Art zu helfen und wandte sich erst an einen Arzt, nachdem sich an der Verletzungsstelle eine weiche Geschwulst von annähernd Kindskopfgrösse (?) eingestellt hatte. Der Kranke wurde dieses Mal nach einem Züricher Privatspital (Nenmünster) verbracht, und hier nahm man eine Eröfnung der Anschwellung vor. Ausser Eiter soll sich ein abgesprengtes Stück einer Rippe aus der Schnittöffnung entleert haben. Die Wunde schloss sich nicht mehr, sondern es blieb eine kleine Oeffnung zurück, aus welcher sich immer etwas röthliche eitrige Flüssigkeit entleerte. Nach einiger Zeit machten sich wieder lebhafterer Husten und reichlicherer Auswurf bemerkbar. Auch wurde Patient während der Nacht 
von sehr lästigen schwächenden Schweissen gepeinigt, und so wurde wieder die Hülfe der medicinischen Klinik nachgesucht.

Die Erkrankung der linken Lunge hatte im Vergleich zu den Veränderungen bei der vorhergegangenen Aufnahme sehr deutliche Fortschritte gemacht. Die Dämpfung über der linken Lunge nahm vorn nicht nur die Supraclaviculargrube, sondern auch die innere Hälfte des ersten linken Intercostalraumes ein, und hinten hatte sich die Dämpfung von der Regio suprascapularis in den oberen Theil des Interscapularraumes bis zur Schultergrube ausgedehnt. Dabei bekam man im Bereiche der Dämpfung Bronchialathmen und reichliche mittelgrosse klingende Blasen zu hören. Der Auswurf war reichlich, eitrig, von röthlich-bräunlicher Farbe und enthielt zahlreiche Tuberkelbacillen. Es zeigte sich ausserdem noch im zweiten linken Intercostalraum in geringer Entfernung vom Brustbein eine ungefähr $7 \mathrm{~cm}$ lange strahlig eingezogene Narbe, in deren oberem besonders stark eingezogenem Rande eine feine Fistelöffnung zu erkennen ist. An dieser Stelle ist die Haut mit dem unteren Rande der ersten linken Rippe unversehieblich verwachsen. Aus der Fistelöffnung entleert sich in mässig reichlicher Menge eine röthlich - eitrige Flüssigkeit, welche in ihrem Aussehen vollkommen an die Beschaffenheit des Auswurfes erinnert, jedoch lassen sich aus ihr keine Tuberkelbacillen gewinnen. Die überraschend grosse Aehnlichkeit zwischen Auswurf und Secret der Fistel legen zwar den Gedanken nahe, dass eine Verbindung zwischen der äusseren Fistelöffnung und der linken Lunge und ihren Bronchien besteht, aber es gelingt nicht, dieselbe mit einiger Sicherheit nachzuweisen. Beim Sondiren der Fistel stösst man sehr bald, entsprechend dem unteren Rande der ersten linken Rippe auf rauhen Knochen. Luft dringt weder bei tiefen Einathmungen in die Fistel hinein, noch bei lebhaften Hustenstössen aus derselben heraus. Milch und dünne Anilinfarbenlösungen, welche man in die Fistelöfnung einspritzt, kommen nicht im Auswurf zum Vorschein. Der Patient erhielt auch dieses Mal wieder Pillen ans Eisen, Bromkali und Arsen und nahm binnen 8 Wochen an Körpergewicht um 2 Kilo zu. Die Wunde hatte man mit Jodoformgaze verbunden, obne dass eine wesentliche Veränderung bemerkbar wurde. Patient wollte wieder den Versuch machen, seine Arbeit aufzunehmen.

Am 13. December 1892 kehrte er jedoch wieder in das Spital zurück. Seine Körperkräfte hatten ausserhalb des Krankenhauses bedeutend abgenommen. $\mathrm{Da}$, wo früher vorn über der linken Lunge Dämpfung gewesen war, hatte sich ein tympanitisch-gedämpfter Percussionsschall eingestellt und bei der Auscultation hörte man hier amphorisehes Athmen und bei der Inspiration zahlreiche grosse metallisch klingende Blasen. An Stelle des früheren Infiltrates hatte sich also eine grössere Höhle ansgebildet, welche von der linken Lungenspitze beginnend die ganze innere Hälfte des ersten linken Intercostalraumes einnahm. Patient hustet grosse Mengen eines vorwiegend eitrigen geballten Auswurfes aus, welcher sebr reich an Tuberkelbacillen ist. 
Die Fistelöffnung im zweiten linken Intercostalraum hat sich nicht geschlossen und ist im Gegentheil grösser als früher. Sie sondert noch immer in unveränderter Weise eine röthlich-bräunliche eitrige, ziemlich dünne Flüssigkeit ab, die sich bei wiederholten Untersuchungen frei von Tuberkelbacillen erweist and nur äusserst sparsame Kokken in baufenförniger Anordnung enthält. Aussaaten von Näbrgelatine blieben steril.

Als ich den Patienten bei der ersten Morgenvisite eingehender untersuchte, erfolgte zufällig bei Entfernung des Verbandes von der Fistelöffnung ein sehr kräftiger Hustenstoss. Zu gleicher Zeit fuhr unter einem zischenden and leicht pfeifenden Geräusche, welches mehrere Schritte weit zu vernehmen war, Luft aus der Fistel heraus, und als man darauf den Kranken auforderte, mehrmals kräftig zo husten, zeigte sich die Erseheinung immer wieder von Neuem, sobald die Kraft der Hustenstösse einen gewissen Werth erreicht hatte. Sehr häufig kam das eitrige Fistelsecret in Form von grösseren Laftblasen mit der Luft aus der Fistel zum Vorschein. Niemals hörte man ein ähnliches Luftgeräusch, wenn der Patient tiefe Einathmangen ausfïhrte.

Die äussere Oefinung der Fistel war in zweiten linken Intercostalraum $8 \mathrm{~cm}$ vom linken Sternalrand gelegen und erreichte den Umfang einer halben Linse. Sie lag nur sebr wenig von dem unteren Rande der ersten Rippe entfernt. Eine Sonde vermochte man nur in der Richtung nach oben und innen gegen das linke Sterno-Claviculargelenk vorzuschieben. In der Regel stiess man mit dem Sondenkopf in einer Tiefe ron $4 \mathrm{~cm}$ auf rauhen Knocheu, welcher der unteren Hälfte der ersten linken Rippe angehörte. Ab und zu gelang es jedoch, die Sonde $6 \mathrm{~cm}$ tief vorwärts zu bewegen, wobei man den Widerstand eines schwammigen weichen Körpers zu empfinden glaubte.

Patient verblieb leider nur 11 Tage im Spital. Obschon sein Leiden, wie bei der früheren Aufnahme, fieberlos verlief, sanken seine Kräfte znsebends, so dass der Kranke, wie er selbst angab, lieber in seinem Hause sterben wollte.

An der so eben beschriebenen vollständigen Lungenhautfistel liess sich ohne besondere Schwierigkeit der Nachweis führen, dass sie einen ventilartigen Bau haben musste, welcher zwar der Luft einen Austritt nach aussen gestattete, es dagegen unmöglich machte, dass in entgegengesetzter Richtung Luft von aussen durch die Fistel in die Lunge eindrang. Wie bereits hervorgehoben wurde, hörte man das Luftgeräusch immer nur während der Exspiration. Freilich musste man zunächst noch den Nachweis liefern, dass überhaupt die äussere Fistelöffnung mit der Lunge und den Bronchialwegen in unmittelbarer Verbindung stand, denn es wäre sehr wohl denkbar gewesen, dass 
ohne eine solche Communication während der Inspiration Luft nur in den äusseren Fistelgang von aussen eingesogen worden wäre, welche alsdann bei kräftigen Hustenstössen unter hörbaren Geräuschen wieder herausgestossen wurde. Von vornherein war es wenig wahrscheinlich, dass man dadurch zum Ziele gelangte, dass man vorsichtig Milch oder gefärbte Flüssigkeiten in die Fistel einspritzte und aufpasste, ob dieselben im Auswurfe wiedererschienen. Eine Fistel mit ventilartigem Verschluss während der Einathmung musste selbstverständlich das Gelingen eines derartigen Versuches vereiteln. Und in der That fielen alle Injectionsversuche ergebnisslos aus. Man suchte sich nun zunächst in folgender Weise zu helfen: Patient wurde aufgefordert mehrmals hinter einander zu husten und gegen Ende des letzten Hustenstosses setzte man fest die Kuppe des angefeuchteten Daumens Iuftdicht auf die Fistelöffnung auf. Bei den tiefen Einathmungen, welche den Hustenstössen folgten, und zu denen der Kranke noch ausdrücklich aufgefordert war, fühlte man nicht den leisesten Aspirationszug am Daumen. Fing nun der Kranke wieder stark zu husten an, ohne dass man zuvor den Daumen von der Fistelöffinung abgehoben hatte, und entfernte nun plötzlich während eines kräftigen Hustenstosses den Daumen von der Fistelöffnung, so hörte und sah man sofort wieder Luft aus der Fistelöffinung nach aussen fabren. Nach dem Gesagten konnte diese Luft begreiflicherweise nur aus den Lungen herstammen, und das Fehlen jeder Ansaugung des Daumens während der Inspiration sprach ausserdem noch für das Bestehen einer Ventilfistel. Noch sicherer konnte der Beweis mit Hülfe eines Quecksilbermanometers geführ' werden, dessen einer Schenkel vermittelst Gummischlauches und eines Metallrohres luftdicht mit der Fistel in Verbindung gebracht wurde. Zum Eindichten der Metallcanüle erwies sich eine Mischung von Wachs, Oel und Lanolin als sebr vortheilhaft, welche sich mittelst Wundwatte, die in eine 0,1 procentige Sublimatlösung getaucht war, rings um die Canüle in der Fistclöffnung anbringen und durch genügend starken Druck zu einem luftdichten Verschlussmittel, das man jeden Augenblick mit Leichtigkeit wieder zu entfernen und dann wieder anzubringen vermochte, umgestalten liess. Hatte nach vorgenommener Ein- 
dichtung die Quecksilbersäule des Manometers den Stand auf dem Nullpunkt eingenommen, so konnte der Kranke durch Hustenstösse das Quecksilber beträchtlich in die Höhe treiben, und die Säule bewahrte unbeeinflusst von allen weiteren Athmungsbewegungen so lange unverändert ihren Stand, bis die Dichtung der Canüle entfernt war.

Gehen wir auf die Grösse der Druckwerthe, welche bei forcirten Ausathmungen (Hustenstössen) an dem Manometer abgelesen werden konnten, etwas genauer ein, so sehen wir dieselben von $10-50 \mathrm{~mm}$ Quecksilber schwanken. Ein Druck der exspiratorischen Lungenluft unter $10 \mathrm{~mm}$ Quecksilber war nicht im Stande, eine Verbindung zwischen den Luftwegen und der äusseren Fistelöffnung herzustellen, so dasss also auch bei der Exspiration erst gewisse Hindernisse überwunden sein mussten, bevor die Lungenluft durch die Fistelöffnung entweichen konnte. Aus diesem Umstand erklärt es sich wohl auch zur Genüge, dass die Werthe, welche in dem Manometer in der Fistelöffnung abgelesen wurden, stets kleiner ausfielen als die Grösse des exspiratorischen Luftdruckes, welcher mit Hülfe eines Waldenburg'schen Pneumatometers in einem Nasenloch bestimmt wurde. Hatte man bei dem Kranken den einen Schenkel eines Waldenburg'schen Manometers mittelst Gummischlauches und Olive luftdicht in ein Nasenloch eingesetzt, während man das andere Nasenloch fest verschloss, so zeigten sich bei ruhiger Athmung folgende Werthe:

$$
\begin{aligned}
& \text { Inspiration }=22 \mathrm{~mm} \mathrm{Hg}, \\
& \text { Exspiration }=44 \mathrm{~mm} \text { Hg. }
\end{aligned}
$$

Bei kräftigen Hustenstössen dagegen konnte der Exspirationsdruck bis $72 \mathrm{~mm} \mathrm{Hg}$ in die Höhe gehen. Begreiflicherweise wäre es sehr unterrichtend gewesen, zu gleicher Zeit und bei den gleichen Hustenstössen Manometermessungen in der Lungenhautfistel und in der Nasenöffnung vorzunehmen, aber leider zeigte sich unser Kranke allen Unterweisungen als wenig zugänglich und scheiterten alle unsere Bemühungen in dieser Richtung.

Nicht ohne Absicht habe ich es versäumt, auf die Literatur der Lungenhautfisteln einzugehen, weil dieselbe erst kürzlich an einem Jedermann leicht zugänglichen Orte von Tschisto- 
witsch ${ }^{1}$ ) wiedergegeben worden ist. Jedoch möchte ich mir die kleine ergänzende Bemerkung erlauben, dass in der so eben erwähnten Zusammenstellang eine Beobachtung von Martel ${ }^{2}$ ) übersehen worden ist.

Zum Schluss ist es mir noch eine angenehme Pflicht, meinem Collegen, Herrn Professor Gaule für seine freundliche Unterstützung bei Ausführung der manometrischen Versuche bestens zu danken.

\section{XV. \\ Zur Localisation der Aphasien.}

Aus der medicin. Klinik des Herrn Prof. Eichhorst in Zürich.

Von Dr. med. J. Leva, Secundararzt der medicin. Klinik in Zürich.

Ueber kein anderes Gebiet der Hirnphysiologie und -Pathologie ist in den letzten 3 Decennien mehr geschrieben worden, als über die Sprache und deren Störungen, speciell über die Aphasie. Die Durchmusterung der endlosen Zahl von veröffentlichten Aphasiefällen ist eine Arbeit, an die sich schon viele und darunter die besten Männer der Wissenschaft gemacht haben; die Ausbeute jedoch für die Förderung unseres Wissens entsprach wegen der Mangelhaftigkeit der Mittheilungen nicht immer der angewendeten Mähe. Nichtsdestoweniger haben Forscher, wie Kussmaul, Wernicke, Lichtheim, Grashey, Charcot, $\mathrm{Naunyn}$ und Andere theils an Hand eigener, genau beobachteter Fälle, theils wo solche, d. h. eben positive Thatsachen, fehlten, durch geistreiche theoretische Erwägungen, uns genaue Pläne vorgezeichnet und gewaltige Bausteine zusammengetragen zu

1) N. Tschistowitsch, Tuberculöse, nach aussen durchgebrochene Caverne (Berlin. klin. Wochenschr. 1892 S. 476.)

2) Marte1, Fistule broncho-cutanée spontanée, mort par infection purulente (Lyon méd. 1875. No. 3. - Vgl. Virchow-Hirsch's Jahresb. 1875. Bd. II. S. 444.) 